\title{
A new method of haemodynamic assessment of mitral stenosis in atrial fibrillation: construction of
}

\section{a nomogram}

\author{
S W Davies, J E Gardener, T J Bowker, A D Timmis, R Balcon
}

\begin{abstract}
Accurate haemodynamic assessment of mitral stenosis by hydraulic formulas requires measurement of the mean valve gradient and the cardiac output. The calculation is laborious, particularly in the presence of atrial fibrillation when averaged values obtained from multiple beat-to-beat determinations must be used. The relations between valve area, end diastolic gradient, and heart rate in 20 patients with mitral stenosis and atrial fibrillation were examined. In each patient the end diastolic pressure gradient for each cardiac cycle was related linearly to the $R R$ interval of that cycle, and this relation was unchanged on exercise. The slope (S) and intercept (I) of this relation correlated with the degree of mitral stenosis as measured by the Gorlin valve area. The regression equations describing these relations were then used to construct a nomogram relating end diastolic pressure gradient to mitral valve area at different heart rates. When the nomogram was applied to catheterisation data from a further $\mathbf{3 0}$ patients the results correlated well with direct calculation of valve area by the Gorlin formula. The nomogram is simple to use, does not require measurement of cardiac output, and is independent of heart rate so that it is unnecessary for the patient to exercise during catheterisation.
\end{abstract}

Haemodynamic assessment of the severity of mitral stenosis from cardiac catheterisation data is time consuming, particularly for patients in atrial fibrillation. The most accurate methods use a hydraulic formula such as that of Gorlin and Gorlin, ${ }^{1}$ which requires measurement of the cardiac output and the mean mitral valve gradient over 10 consecutive cardiac cycles (by planimetry or from digitised signals). In practice many cardiologists rely on the end diastolic gradient across the mitral valve..$^{2-5}$ Although it is recognised that this is highly dependent upon heart rate, ${ }^{6-9}$ there are no published tables relating end diastolic gradient, heart rate, and the severity of stenosis. We therefore examined the relation between these variables in order to devise a method of estimating the severity of mitral stenosis and the mitral valve area from the simple measure of end diastolic gradient. A simple nomogram was constructed and the reliability of this approach was assessed in another group of patients.

\section{Patients and methods}

PATIENTS

Cardiac catheterisation data from an initial group of 20 patients were analysed in detail. All 20 were judged to have rheumatic valve disease on the basis of a past history of rheumatic fever and typical $M$ mode echocardiographic features and all were in established atrial fibrillation.

To validate the nomogram constructed on the basis of the first 20 patients, we analysed cardiac catheterisation data in a further 30 patients. All had moderate or severe mitral stenosis; 25 were in established atrial fibrillation and five remained in sinus rhythm at the time of the investigation.

\section{MEASUREMENTS}

All patients were catheterised by the Sones technique, with an 8 French Sones catheter positioned in the body of the left ventricle and a 7 French Cournand catheter advanced to wedge in an upper lobe division of either the right or left pulmonary artery. The electrocardiogram, pulmonary artery wedge pressure, and the left ventricular pressure were recorded simultaneously at high paper speed of $100 \mathrm{~mm} / \mathrm{s}$ and high sensitivity with careful calibration and balancing. In each patient 50 consecutive cardiac cycles were recorded at rest, while the patient was supine on the cardiac catheterisation table. In 10 patients who were able to perform supine straight leg raising exercises sufficient to increase mean heart rate by $\geqslant 20 \%, 50$ consecutive cycles were also recorded immediately after this exercise. Cardiac output at rest was calculated by the indirect Fick method. The mitral valve area was calculated by the formula of Gorlin and Gorlin, ${ }^{1}$ modified to take account of left ventricular pressure measured directly. ${ }^{10}$

The $R R$ interval was measured from the peak of intrinsicoid QRS deflection to the peak of the intrinsicoid deflection of the next QRS complex; extrasystoles were included in 
Figure 1 Measurement of mitral valve gradient at end diastole and $R R$ interval for each cardiac cycle.
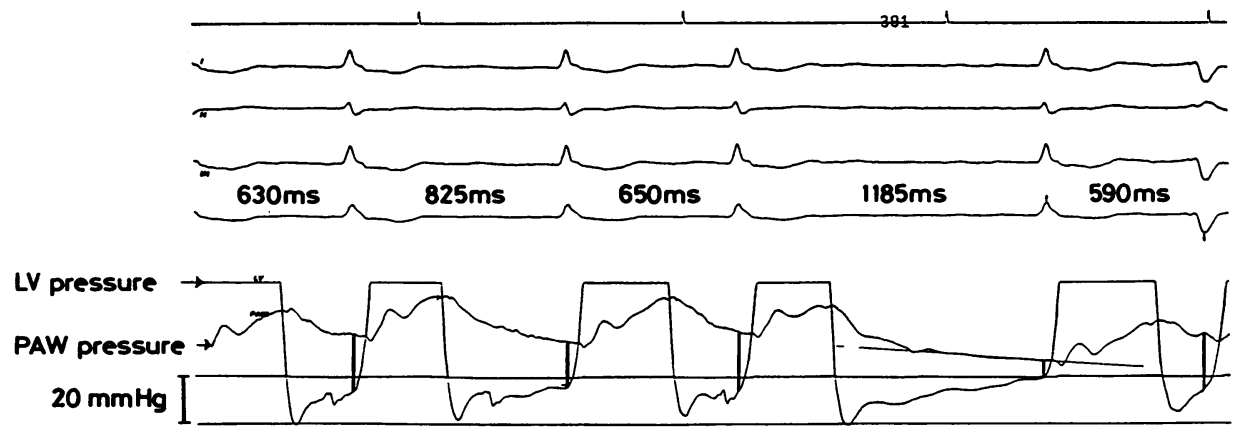

is

the analysis. The end diastolic gradient across the mitral valve was measured at the point of upward inflection in the left ventricular pressure trace or, if there was no well defined inflection, was taken at the peak of the intrinsicoid deflection of the QRS complex (see fig 1).

\section{ANALYSIS}

The relation between the end diastolic gradient across the mitral valve and $R R$ interval in each subject was analysed by linear regression over the range of $R R$ intervals at which the end diastolic gradient was greater than zero. The goodness of fit in each patient was judged by the Pearson product-moment correlation coefficient. When subjects were compared a normal distribution could not be assumed in view of the small numbers (20), and so we used the appropriate distributionfree statistic (Spearman rank correlation coefficient $\mathbf{R}$ ).

\section{Results}

RELATION OF END DIASTOLIC GRADIENT TO RR INTERVAL

The range of $R R$ intervals in each patient was (median) 470-1250 ms-the narrowest range being $550-1080 \mathrm{~ms}$ and the widest range 240 $1875 \mathrm{~ms}$. The relation between the end diastolic gradient across the mitral valve and RR interval was monotonic, with the end diastolic gradient decreasing as RR interval lengthened (Spearman $R>0.8$ in each of 20 patients) until a critical RR interval above which left ventricular and pulmonary artery wedges

Figure 2 Examples of the relation between mitral valve gradient at end diastole and $R R$ interval in two patients.

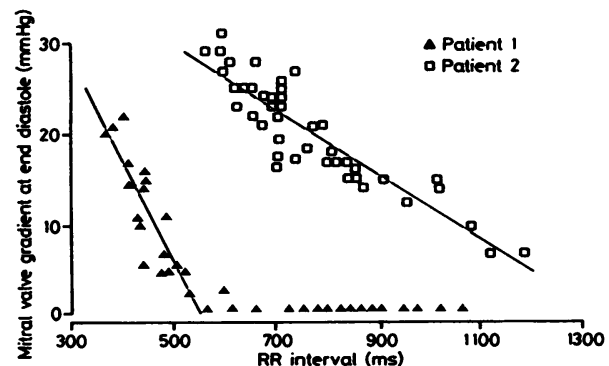

equilibrated and the end diastolic gradient was zero (fig 2). At RR intervals shorter than this critical $R R$ interval the relation was approximately linear, and for each patient linear regression analysis was used to obtain the best fitting equation over this range. Goodness of fit judged by the Pearson correlation coefficient $r$ over this range of $R R$ was $>0.8$ in each individual patient.

Supine exercise performed in 10 patients so as to increase mean heart rate by $\geqslant 20 \%$ over the resting state did not seem to influence the relation of end diastolic gradient to $R R$ interval (fig 3). However, there was considerable scatter in the data points so that a given $R R$ interval was associated with a range of end diastolic gradients. It is therefore possible that changes in the relation occur on exercise but are obscured by this variation.

CORRELATIONS WITH MITRAL VALVE AREA There were significant correlations between the severity of mitral stenosis as measured by the mitral valve area calculated according to the Gorlin formula and the following variables:

(a) The slope of the relation of end diastolic mitral valve gradient to $R R$ interval correlated with the Gorlin mitral valve area $\left(\mathrm{MVA}_{\mathrm{G}}\right)$ for that patient (fig 4). The Spearman correlation coefficient was $R=+0.88(p<0.005)$ and the linear regression equation was:

$$
\text { slope }=10 \cdot 4\left(\mathrm{MVA}_{\mathrm{G}}\right)-1 \cdot 7 .
$$

(b) The intercept of the relation of end diastolic mitral valve gradient to $R R$ interval correlated with the Gorlin mitral valve area for that patient (fig 5). The Spearman correlation coefficient was $R=-0.90(p<0.005)$ and the linear regression equation was:

$$
\text { intercept }=1460-662\left(\mathrm{MVA}_{\mathrm{G}}\right) \text {. }
$$

Possible covariables in this analysis include age, height, and weight. However, the Gorlin mitral valve area was not found to be a function of age, height, or weight in this group of subjects. It is therefore unlikely that they confounded the analysis of $\mathrm{MVA}_{\mathrm{G}}$ with respect to the slope or intercept.

\section{NOMOGRAM}

The above regression equations enabled the construction of a nomogram relating end diastolic mitral valve gradient to the Gorlin valve 
Figure 3 Relation between mitral valve gradient at end diastole and $R R$ interval before and after exercise in one patient.

Figure 4 (A) Slope and (B) intercept of the mitral valve gradient at end diastole ( $M V G-E D)$ relation to $R R$ interval and enclosed area plotted against the Gorlin mitral valve area.
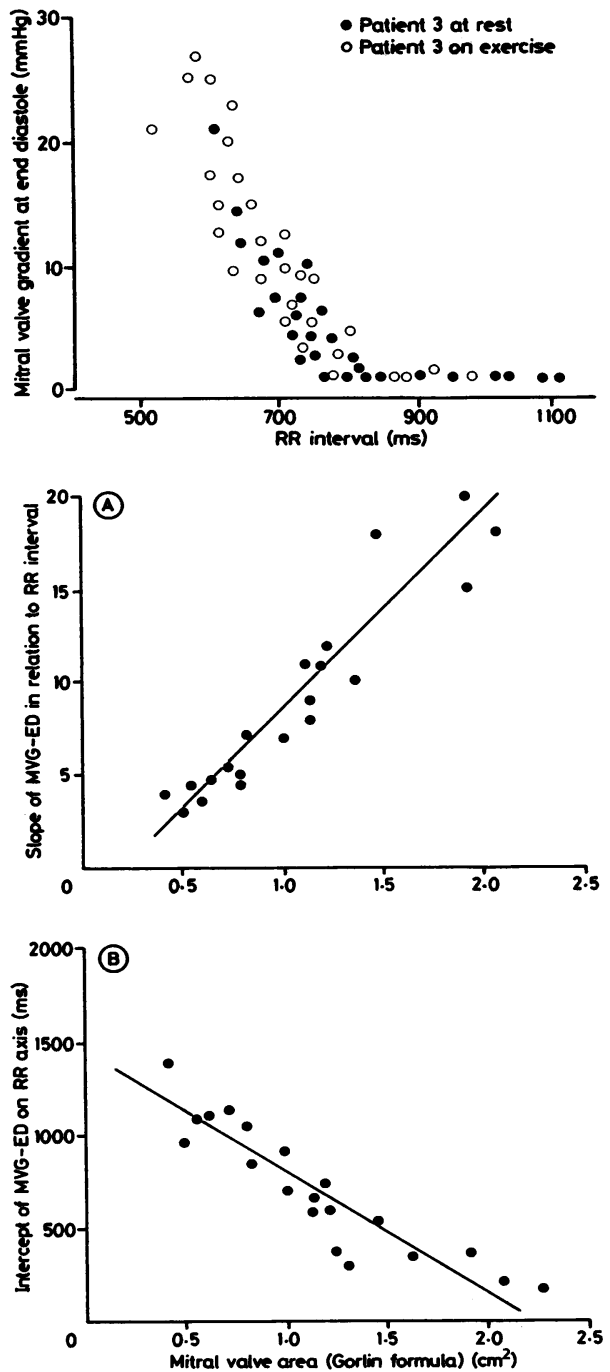
predict Gorlin valve area
Figure 5 Nomogram to from mitral valve area at end diastole and $R R$ interval or heart rate.

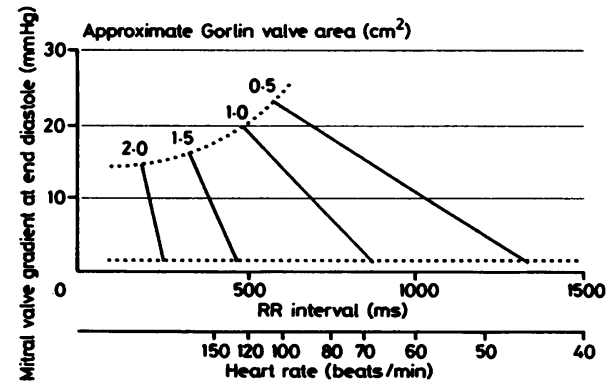

Mitral valve areas predicted by nomogram and directly calculated in 30 patients with mitral stenosis

\begin{tabular}{llllll}
\hline \multirow{2}{N}{$\begin{array}{l}\text { Nomogram } \\
\text { prediction }\left(\mathrm{cm}^{2}\right)\end{array}$} & \multicolumn{5}{l}{ Gorlin mitral valve area $\left(\mathrm{cm}^{2}\right)$} \\
\cline { 2 - 6 } & $<0.5$ & $0.5-1.0$ & $1.0-1.5$ & $1.5-2.0$ & $>2.0$ \\
\hline$>2.0$ & & 3 & 1 & 3 & \\
$1.5-2.0$ & 14 & 6 & & \\
$1.0-1.5$ & 2 & & & & \\
$0.5-1.0$ & $<0.5$ & & & & \\
\hline
\end{tabular}

area at a range of different heart rates. Chosen values of Gorlin valve area $(2.0,1.5,1.0$, and $0.5 \mathrm{~cm}^{2}$ ) were substituted in the equations, and the predicted lines relating end diastolic mitral valve gradient to $R R$ interval were plotted in fig 5. Broken lines indicate the limits beyond which there are insufficient data to extrapolate these relations.

The nomogram permitted rapid estimation of valve area-for example the data in fig 1 show a cardiac cycle with RR interval $825 \mathrm{~ms}$ and an end diastolic gradient of $18 \mathrm{~mm} \mathrm{Hg}$. On the nomogram the estimated valve area lies between $0.5 \mathrm{~cm}^{2}$ and $1.0 \mathrm{~cm}^{2}$. Direct application of the Gorlin formula to the catheterisation data gave a valve area of $0.68 \mathrm{~cm}^{2}$ in this patient. All the other cardiac cycles in this recording gave a similar estimate from the nomogram.

The nomogram was validated by analysis of cardiac catheterisation data for a further $\mathbf{3 0}$ patients with mitral stenosis (five in sinus rhythm and 25 in atrial fibrillation). The Gorlin valve area was first predicted from the nomogram by plotting the mitral valve gradient at end diastole against the RR interval for each of between two and five cardiac cycles. The valve area was then calculated directly from the indirect Fick cardiac output and 10 planimetered cardiac cycles by the Gorlin and Gorlin formula. In 25 out of $\mathbf{3 0}$ cases there was agreement, and in only one case was there a significant discrepancy (table).

\section{Discussion}

The dependence of the end diastolic mitral valve gradient on heart rate (and on $R R$ interval) has long been appreciated..$^{6-9}$ It may be particularly difficult to interpret the end diastolic gradient at extremes of heart rate ${ }^{11}$ and in patients in atrial fibrillation. Whereas corrections have been described for the effect of heart rate on the peak to peak aortic valve gradient, ${ }^{12}$ none has been formulated for the mitral valve gradient. Accordingly, supine exercise during cardiac catheterisation has for many years been advocated as a means of identifying patients with important mitral stenosis who at rest have a normal or slow heart rate with only small to moderate transmitral gradients. ${ }^{69}$ However, exercise during cardiac catheterisation is often hindered by patient discomfort, poor motivation, and the need to maintain sterility. ${ }^{9}$ Increased ventilation by the patient may cause wide swings in intrathoracic pressure, confusing the interpretation of the pressure tracings. ${ }^{9}$ In those patients unable to increase their heart rate sufficiently by exercise during catheterisation it has been suggested that atropine or dobutamine should be used. ${ }^{13} 14$ The converse problem may arise in patients who are anxious or unwell at the time of catheterisation in whom it is not always possible or desirable to slow a fast heart rate. The effects of extreme heart rates on the assessment may unduly influence the choice between medical treatment and valve replacement or balloon dilatation if the severity of mitral stenosis is borderline.

The problem of differing heart rates in the 
assessment of mitral stenosis can be partly avoided by applying hydraulic formulas to catheterisation data to calculate the effective area of the valve orifice. The best known and most widely used of the hydraulic formulas is that developed by Gorlin and Gorlin on the basis of Poiseuille's law and Torricelli's orifice equation. ${ }^{1}$ This has the general form:

valve area $=\frac{\text { mean flow rate through valve }}{\text { empirical constant } \times \sqrt{ } \text { mean pressure difference }}$

the empirical constant being different for mitral stenosis and for aortic stenosis. ${ }^{1}$ Modifications to the original formula ${ }^{15-18}$ and even new hydraulic formulas ${ }^{19-21}$ have been proposed, but the Gorlin formula remains the most widely used. ${ }^{45}$ Estimates of mitral valve area by this method correlate well with valve area determined at surgery, ${ }^{1}$ although there may be a tendency systematically to underestimate valve area under conditions of very low cardiac output. ${ }^{22}$ In the present study, resting cardiac output was $\geqslant 2.51 / \mathrm{min}$ in all cases (mean $3.41 / \mathrm{min}$ ), so the Gorlin formula is likely to have provided an accurate estimate of valve area.

Non-invasive assessment of the severity of mitral stenosis by conventional or Doppler echocardiography or both is convenient and reliable. ${ }^{24-26}$ However, echocardiography. requires a skilled operator and clear recordings are not possible in up to $10 \%$ of patients. ${ }^{27-29}$ Care is needed in interpretation because these methods may be inaccurate at fast heart rates and at high flows ${ }^{30}$ and when the valve orifice is very small. ${ }^{31}$ Mitral pressure half time in particular is influenced by several factors besides valve area. ${ }^{2132}$ Thus in patients undergoing cardiac catheterisation for another reason, such as coexisting coronary artery disease, the haemodynamic data obtained may be useful in assessing the severity of mitral stenosis.

Hydraulic formulas arguably provide the most accurate estimates of valve area, ${ }^{15}$ but are tedious to use and require measurement of cardiac output. The labour can be reduced by digitisation of pressure signals to enable computed calculation, but such facilities are not widely available. The time taken for accurate planimetry led Potanin et al ${ }^{33}$ to suggest superimposition of pressure tracings and visual comparison of areas as an alternative. However, like the nomogram of Negri et $a^{l^{4}}$ (based on a simplified hydraulic formula rather than the Gorlin formula) this approach still requires the cardiac output to be measured. A nomogram relating the end diastolic mitral valve gradient and heart rate to valve area might allow accurate assessment more conveniently than either Gorlin's original formula or Negri's nomogram.

Our data suggest that the relation between the end diastolic mitral valve gradient and $R R$ interval is approximately linear. Regression analysis provides an equation for each patient that fits the data points well with correlation coefficient $r>0.8$, provided that care has been taken to obtain a technically satisfactory pulmonary wedge trace with little respiratory variation in the pressure. This linear relation contrasts with the time course of the left ventricular-pulmonary wedge pressure difference during diastole in each individual cardiac cycle, when the pressure difference seems to decline in an approximately exponential fashion. The explanation for this discrepancy is uncertain - it may depend on interactions between the length of preceding cardiac cycles and haemodynamic factors including left atrial filling ${ }^{35}$ and the inotropic ${ }^{36-38}$ and lusitropic ${ }^{39-41}$ state of the left ventricle, which may be particularly complex in atrial fibrillation. ${ }^{42}$ The simplicity of the linear relation between end diastolic gradient and RR interval is, however, an advantage in the construction of $a$ nomogram (fig 5).

The nomogram is easy to use and a single data point may suffice. Any single cardiac cycle from the recording in fig 1 gave a point between the $0.5 \mathrm{~cm}^{2}$ and $1.0 \mathrm{~cm}^{2}$ lines of the nomogram when the Gorlin calculation gave a value of 0.68 $\mathrm{cm}^{2}$ in that patient. The nomogram was validated by comparison with direct calculation of the valve area by the Gorlin formula in a separate group of 30 patients with mitral stenosis. There was agreement in 25/30 (83\%) and disagreement in five. The largest difference was in a patient with a directly calculated valve area of $2.2 \mathrm{~cm}^{2}$, in whom the nomogram predicted a valve area of between 1.0 and 1.5 $\mathrm{cm}^{2}$. Even this difference, although undesirable, was unlikely to lead to a major change in the management of the individual patient.

The high level of agreement between nomogram estimates and direct Gorlin formula estimates of mitral valve area is perhaps surprising because cardiac output is required for calculation of the Gorlin valve but is not for the nomogram. Estimation of cardiac output is often inaccurate and this might be expected to produce discrepancies between the two methods. However, when the two methods were compared, the estimates of mitral valve area were divided into only five categories (corresponding to clinically very severe, severe, moderate, mild, and trivial mitral stenosis) (table). Thus even a $20-30 \%$ error in the estimation of cardiac output might not change the category of valve area into which the Gorlin formula estimate falls. Obviously in a simple analysis end diastolic gradient must depend upon cardiac output and flow rate across the valve as well as upon heart rate and mitral valve area. Furthermore, mitral valve area is, to a certain extent, influenced by flow rate. The apparent independence of the nomogram from the cardiac output may simply reflect a narrow range of cardiac outputs in the subjects in the study, or a systematic relation between valve area and resting cardiac output, or both.

There are several other sources of inaccuracy in the data from which the nomogram was derived that might reduce the accuracy of the nomogram. These are the use of fluid filled rather than transducer tipped catheters, the use of pulmonary capillary wedge pressure to reflect left atrial pressure, and the use of the indirect Fick method to measure cardiac output.

Another possible cause of error is mitral 
regurgitation, which leads to greater mean diastolic transmitral flow than would be calculated from the net forward cardiac output. This will lead to underestimation of mitral valve area by the Gorlin formula, on which this nomogram is based. ${ }^{2}$ None of the subjects in the present study had mitral regurgitation in excess of angiographic grade 2 : it is unlikely that the nomogram will be applicable in cases with severe mitral regurgitation. Subject to this restriction, the nomogram may be used in any patient in whom a significant end diastolic gradient has been recorded and can be related to the $\mathbf{R R}$ interval.

\section{CONCLUSION}

In mitral stenosis complicated by atrial fibrillation, the linear relation between $R R$ interval and mitral valve gradient at end diastole permits the calculation of simple variables that correlate with mitral valve area calculated by the Gorlin formula. They can thus be used to assess the severity of mitral stenosis, and this is done most conveniently in the form of a nomogram. The nomogram correlates closely with the Gorlin hydraulic formula but is simpler to use and does not require cardiac output measurement. Furthermore, it is independent of heart rate and it is therefore unnecessary for the patient to exercise during cardiac catheterisation.

1 Gorlin R, Gorlin SG. Hydraulic formula for calculation of the area of the stenoic mitral valve, other cardiac valves and central circulatory shunts. I. Am Heart J 1951;41: $1-29$.

2 Selzer A, Willett FM, McCaughey DJ, Feichtmeir TV. Uses of cardiac catheterisation in acquired heart disease. $N$ Engl of cardiac catheterisation in acquire

3 Verel D, Grainger RG. Cardiac catheterisation and angiocardiography. 3rd ed. London: Churchill Livingstone, 1978:11.

4 Mendel D, Oldershaw P. A practice of cardiac catheterisation. 3rd ed. London: Blackwell Scientific, 1986:185 and 240.

5 Hurst JW. The heart. 6th ed. New York: McGraw-Hill, 1986:649 and 759.

6 Wood P. An appreciation of mitral stenosis. $\mathrm{Br}$ Med J 1954;i: $1051-63$ and $1113-24$.

7 Nakhjavan FK, Katz M, Shedrilovzky H, et al. Hemodynamic effects of exercise, catecholamine stimulation and tachycardia in mitral stenosis and sinus rhythm at comparable heart rates. Am J Cardiol 1969;23:659-66.

8 Manchanda SC, Ramesh L, Roy SB. Haemodynamic effects of atrial pacing in rheumatic mitral stenosis. Br Heart $J$ 1974;36:636-40.

9 Grossman $\mathbb{W}$, McLaurin LP. Dynamic and isometric exercise during cardiac catheterisation. In: Grossman W, ed.
Cardiac catheterisation and angiography. Philadelphia: Lea Cardiac catheterisation and

10 Cohen MV, Gorlin R. Modified orifice equation for the calculation of mitral valve area. Am Heart J 1972;84: 839-40.

11 Odemuyiwa O, Hall RJC. Assessing the severity of valve stenosis. Br Heart J 1986;55:117-9.

12 Rozenman Y, Gotsman MS. Heart rate influence on the systolic gradient across the stenotic aortic valve: theoretical evaluation and implications. Cathet Cardiovasc Diagn 1985;11:533-8.

13 Angel J, Domingo E, Soler-Soler J, Anivarro I. Hemodynamic evaluation of stenotic cardiac valves. I. Effect of ventriculography and atropine on mitral stenosis. Cather Cardiovasc Diagn 1985;11:115-25.

14 Hwang MH, Pacold I, Piao ZE, et al. The usefulness of dobutamine in the assessment of the severity of mitral stenosis. Am Heart $J 1986 ; 111: 312-6$.

15 Hammermeister KE, Murray JA, Blackmon JR. Revision of Gorlin constant for calculation of mitral valve area from Gorlin constant for calculation of mitral valve
left heart pressures. Br Heart J 1973;35:392-6.
16 Seitz W, Marino $P$, Zanolla $L$, et al. Cardiac valve orifice equation independent of valvular flow intervals: application to mitral valve area computation and comparison with tion to mitral valve area computation and comparison with the Gorlin formula and direct a
Cardiovasc Res 1984;18:669-74.

17 Cannon SR, Richards KL, Crawford M. Hydraulic estimation of stenotic orifice area: a correction of the Gorlin formula. Circulation 1985;71:1170-8.

18 Gorlin WB, Gorlin R. A generalised formulation of the Gorlin formula for calculating the area of the stenotic mitral valve and other stenotic cardiac valves. J Am Coll Cardiol 1990;15:246-7.

19 Hakki AH, Iskandrian As, Bemis CE, et al. A simplified valve formula for the calculation of stenotic cardiac valve areas. Circulation 1981;63:1050-8.

20 Angel J, Soler-Soler J, Anivarro I, Domingo E. Hemodynamic evaluation of stenotic cardiac valves. II. Modification of the simplified valve formula for mitral and aortic valve area calculation. Cathet Cardiovasc Diagn valve area calcu

21 Thomas JD, Weyman AE. Fluid dynamics model of mitral valve flow: description with in vitro validation. J Am Coll valve flow: description with

22 Segal J, Lerner DJ, Miller DC, et al. When should Dopplerdetermined valve area be better than the Gorlin formula?: variation in hydraulic constants in low flow states. $\mathrm{J} \mathrm{Am}$ Coll Cardiol 1987;9:1294-305.

23 Richter HS. Mitral valve area: measurement soon after catheterisation. Circulation 1963;28:451-4.

24 Hatle L, Brubakk A, Tromsdal A, Angelsen B. Noninvasive assessment of pressure drop in mitral stenosis by Dopple ultrasound. Br Heart $J$ 1978;40:131-40.

25 Graf JH, Meltzer R. Echocardiography in mitral valve disease: a review. Int J Cardiac Imaging 1985;1:189-205.

26 Loperfido F, Laurenzi F, Gimigliano F, et al. A comparison of the assessment of mitral valve area by continuous wave Doppler and by cross sectional echocardiography. $\mathrm{Br}$ Heart $J$ 1987;57:348-55.

27 Reifart N, Baykut D, Nowak B, Satter P. Problems encountered in the echocardiographic quantification of severe mitral stenoses. Z Kardiol 1986;75:463-7.

28 Stamm RB, Martin RP. Quantification of pressure gradients across stenotic valves by Doppler ultrasound. J Am Coll Cardiol 1983;2:707-18.

29 Smith MD, Kwan OL, De Maria AN. Value and limitations of continuous wave Doppler-echocardiography in estimating severity of valvular stenosis. JAMA 1986; 255:3145-51.

30 Sagar KB, Wann LS, Paulson WJH, Lewis S. Role of exercise Doppler echocardiography in isolated mitral stenosis. Chest 1987;92:27-30.

31 Vasko SD, Goldberg SJ, Requarth JA, Allen HD. Factors affecting the accuracy of in vitro valvar pressure gradient estimates by Dof

32 Thomas JD, Weyman AE. Doppler mitral pressure halftime: a clinical tool in search of theoretical justification. $J$ time: a clinical tool in search of the

33 Potanin C, Jackson DH, Burdeshaw J. Determination of valvular pressure gradients by visual comparison of areas. J Appl Physiol 1970;29:717-9.

A, Martuscelli E, Mangieri E, et al. Nomogram for calculation of stenotic cardiac valve areas from cardiac output and mean transvalvular gradient. Cathet Cardiovasc Diagn 1984;10:613-8.

35 Keren G, Meisner JS, Sherez J, et al. Interrelationship of mid-diastolic mitral valve motion, pulmonary venous flow, and transmitral flow. Circulation 1986;74:36-44.

36 Gibson DG, Broder G, Sowton E. Effect of varying pulse interval in atrial fibrillation on left ventricular function in interval in atrial fibrillation on left
man. $B r$ Heart $J 1971 ; 33: 388-93$.

37 Karliner JS, Gault JH, Bouchard RJ, Holzer J. Factors influencing the ejection fraction and the mean rate of influencing the ejection fraction and the mean rate of circumferential fibre shortening during
in man. Cardiovasc Res $1974 ; 8: 18-25$.

38 Pfeiffer KP, Kenner T, Schaefer J. Application of statistical methods for the analysis of interval related cardiac performance variations during cardiac arrhythmia in man. Cardiovasc Res 1984;18:80-98.

39 Sonnenblick EH, Ross J Jr, Covell JW, Braunwald E. Alterations in resting length-tension relations of cardiac muscle induced by changes in contractile force. Circ Res 1966;19:980-8.

40 Covell JW, Ross J Jr, Taylor R, Sonnenblick EH, Braunwald E. Effects of increasing frequency of contraction on forcevelocity relation of left ventricle. Cardiovasc Res 1967;1: 2-8.

41 Lavine SJ, Krishnaswami V, Levinson N, Shaver JA. Effect of heart rate alterations produced by atrial pacing on the pattern of diastolic filling in normal subjects. $\mathrm{Am} \mathrm{J} \mathrm{Cardiol}$ pattern of diastolic fill

42 Rawles JM. A mathematical model of left ventricular function in atrial fibrillation. Int J Biomed Comput 1988;23:57-68. 